\title{
Dependencia a la nicotina y preparación para dejar de fumar en la población española
}

\section{Nicotine dependence and readiness to quit smoking in the Spanish population}

\author{
Marcela Fu*, ${ }^{*},{ }^{* * *}$; Jose M. Martínez-Sán- \\ CHEZ $^{*},{ }^{* \star},{ }^{* * *} ;$ MARÍA J. LÓPEZ ${ }^{\star \star \star *},{ }^{* * * * *}$;

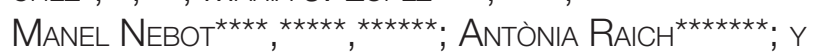 \\ ESTEVE FERNÁNDEZ ${ }^{\star},{ }^{* \star},{ }^{* \star \star}$; \\ EN NOMBRE DEL ETS EUROSURVEY WORKING GROUP\$
}

$\S$ El Environmental Tobacco Smoke (ETS) EuroSurvey Working Group está compuesto por: M. Nebot, M.J.López, F. Centrich, E. Fernández, A. Schiaffino, H. Moshammer, M. Neuberger, G. Gorini, M. Albertini, M. Mulcahy, K. Prezwozniak, W. Zatonski, J. Gumkowski, M. Chudzikova, M. Pilali y P. Birkui.

\author{
Enviar correspondencia a: \\ Marcela Fu \\ Unidad de Control del Tabaquismo, Programa de Prevención y \\ Control del Cáncer, Institut Català d'Oncologia \\ Av. Gran Via de L'Hospitalet, 199-203 \\ 08908 L'Hospitalet de Llobregat (Barcelona) \\ Tel.: 93-2607357; Fax: 93-2607956 \\ E-mail: mfu@iconcologia.net
}

\section{RESUMEN}

Objetivo: Describir la dependencia a la nicotina y la preparación para dejar de fumar en la población fumadora.

Métodos: Estudio transversal de una muestra representativa de la población española $\geq 18$ años. La información se obtuvo mediante entrevistas telefónicas realizadas en junio y julio de 2006. Se estudió la dependencia a la nicotina mediante el test de Fagerström (FTND) y la preparación para dejar de fumar de acuerdo a los estadios del cambio del modelo transteórico en una muestra de fumadores de cigarrillos.

Resultados: El 22,5\% de los participantes (IC 95\%: 20,9-24,2\%) fumaba cigarrillos. Su consumo medio fue de 14,4 cigarrillos diarios (desviación estándar 9,15 ) y su puntuación media en el FTND fue de 2,8 , sin diferencias según los estadios del cambio. El 64,3\% (IC 95\%: 60,3-68,2\%) de los fumadores estaba en el estadio de precontemplación, el 25,4\% (IC 95\%: 21,8-28,9\%) en el de contemplación y el 10,4\% (IC 95\%: 7,9-12,9\%) en el estadio de preparación, sin diferencias por sexo. Los fumadores más dependientes a la nicotina ( FTND $\geq 6$ ) tenian mayoritariamente estudios primarios, comenzaron a fumar a edad más temprana y fumaban más cigarrillos al día.

Conclusiones: Los fumadores presentan mayoritariamente un bajo nivel de dependencia a la nicotina y se encuentran en fase de precontemplación. Los programas de deshabituación deberian ir dirigidos a reducir el nivel de dependencia, ayudar a los fumadores a avanzar a través de los estadios del cambio y, consecuentemente, disminuir la prevalencia de fumadores en la población.

Palabras clave: tabaquismo, dependencia a la nicotina, estadios del cambio, modelo transteórico, prevalencia, epidemiología
Unidad de Control del Tabaquismo, Programa de Prevención y Control del Cáncer, Institut Català d'Oncologia - ICO. Av. Gran Via de L'Hospitalet, 199-203. 08908 L'Hospitalet de Llobregat (Barcelona). Grupo de Prevención y Control del Cáncer, Institut d'Investigació Biomèdica de Bellvitge - IDIBELL. Av. Gran Via de L'Hospitalet, 199203. 08908 L'Hospitalet de Llobregat (Barcelona).

*** Departamento de Ciencias Clínicas, Universitat de Barcelona Campus de Bellvitge. Pavelló de Govern, Feixa Llarga s/n. 08907 L'Hospitalet de Llobregat (Barcelona).

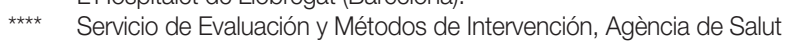
Pública de Barcelona (ASPB). PI. Lesseps 1. 08023 Barcelona. CIBER de Epidemiología y Salud Pública (CIBERESP). Parc de Recerca Biomèdica de Barcelona, Doctor Aiguader $881^{\text {a }}$ Planta. 08003 Barcelona.

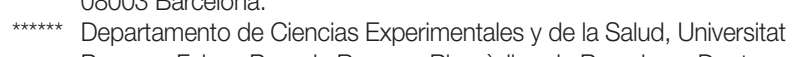
Pompeu Fabra. Parc de Recerca Biomèdica de Barcelona, Doctor Aiguader 88. 08003 Barcelona.

* Althaia Red Asistencial de Manresa. Dr. Llatjos, s/n Edifici CSAM. 08243 Manresa (Barcelona).

\section{ABSTRACT}

Objective: To describe the nicotine dependence and readiness to quit smoking in the smoker population.

Methods: Cross-sectional study on a representative sample of the Spanish population of $\geq 18$ years old. We gathered information by means of telephone interviews conducted between June and July of 2006. We studied the nicotine dependence with the Fagerström Test for Nicotine Dependence (FTND) and the readiness to quit according to the stages of change from the Transtheoretical Model in a sample of cigarette smokers. Results: $22.5 \%$ of participants (95\% Cl: 20.9-24.2\%) smokers cigarettes. They smoked an average of 14.4 cigarettes per day (standard deviation 9.15) and the mean FTND score was 2.8, with no differences by the stages of change. $64.3 \%$ (95\% Cl: $60.3-68.2 \%)$ of smokers were in the precontemplation stage, $25.4 \%$ (95\% Cl: 21.8-28.9\%) in contemplation, and $10.4 \%$ (95\% Cl: $7.9-12.9 \%)$ in preparation, with no differences by sex. The most nicotine dependent smokers (FTND $\geq 6$ ) had mainly primary studies, started to smoke at earlier ages, and smoked more cigarettes per day.

Conclusions: Most Spanish smokers have low nicotine dependence and are in precontemplation stage. Smoking cessation programmes should be addressed to reduce dependence, help smokers to progress through the stages of change, and, consequently, reduce the prevalence of smokers in the population.

Keywords: smoking, nicotine dependence, stages of change, transtheoretical model, prevalence, epidemiology 


\section{INTRODUCCIÓN}

E I consumo de tabaco aumenta el riesgo de padecer enfermedades respiratorias agudas y crónicas, así como enfermedades cardiovasculares, cáncer y alteraciones de la función reproductiva ${ }^{1}$. Informar a los fumadores acerca de los efectos nocivos del tabaquismo para la salud y estimularlos a abandonar el consumo son objetivos fundamentales en la práctica clínica en particular y de la salud pública en general. Para poder desarrollar programas de prevención e intervención orientados a cumplir estos objetivos resulta de gran interés conocer el nivel de dependencia a la nicotina y la preparación de los fumadores para dejar de fumar.

Existe una serie de instrumentos para evaluar la dependencia a la nicotina. Uno de los más utilizados en el ámbito clínico es el test de Fagerström de dependencia a la nicotina ${ }^{2,3}$, debido a su brevedad y sencillez de aplicación. Por otra parte, para evaluar la preparación para dejar de fumar en el contexto de la deshabituación tabáquica se ha utilizado ampliamente el modelo transteórico de Prochaska y DiClemente ${ }^{4}$, que es un modelo integrado de cambio conductual basado en el proceso de toma de decisiones realizado por un individuo. Según este modelo, el proceso de cambio tiene lugar a través de 5 etapas o estadios, en cuyo camino el fumador avanza progresivamente hasta dejar el hábito de fumar.

En España existen algunos estudios que han descrito la dependencia a la nicotina o la preparación para dejar de fumar ${ }^{3,5-11}$. Sin embargo, la mayoría de ellos se han realizado en un contexto clínico o de cesación tabáquica ${ }^{7-9,11}$ y ninguno de los estudios realizados a nivel poblacional incluyó una muestra representativa de la población adulta española ${ }^{3,5,6,10}$. El estudio de la dependencia y la preparación para dejar de fumar a nivel poblacional puede ser útil para desarrollar y evaluar políticas públicas dirigidas a disminuir el consumo y fomentar la cesación tabáquica. El objetivo del presente trabajo es describir la dependencia a la nicotina y la preparación para dejar de fumar (estadios del cambio) en la población adulta española.

\section{MÉTODOS}

Los datos provienen de un estudio multicéntrico de diseño transversal con el objetivo de describir la prevalencia y algunas características del consumo de tabaco (como la dependencia a la nicotina y los estadios del cambio) y la exposición al humo ambiental del tabaco en la población general de 6 países europeos ${ }^{12}$. Este trabajo está centrado en los datos de España ${ }^{13}$, cuya muestra teórica de 2.500 personas se obtuvo de forma proporcional por comunidad autónoma, tamaño del municipio de residencia, sexo y grupos de edad. El muestreo contempló la sustitución de las no-respuestas a partir de los mismos estratos. Los participantes fueron seleccionados en dos fases: en la primera de ellas se escogieron hogares de manera aleatoria a partir de un directorio telefónico, y en la segunda fase se seleccionó a la persona del hogar que respondería a la encuesta, de acuerdo a los estratos establecidos en el muestreo. Después de otorgar el consentimiento oral, los participantes fueron encuestados telefónicamente por entrevistadores entrenados durante junio y julio de 2006. La muestra final, representativa de la población adulta española $\geq 18$ años, estuvo conformada por 2.538 personas y se obtuvieron 2.522 encuestas válidas (1.221 hombres y 1.301 mujeres). El 23,4\% eran fumadores $(22,0 \%$ fumadores diarios y $1,4 \%$ fumadores ocasionales), el $27,3 \%$ eran exfumadores y el $49,2 \%$ de los participantes no había fumado nunca.

\section{Variables}

Se definió como fumadores a aquellas personas que declararon fumar cigarrillos diaria u ocasionalmente en el momento de realizar la entrevista $(n=568)$. No fueron incluidas en el análisis aquellas personas que sólo fumaban puros o cigarros, puritos, pipas o tabaco de liar $(n=23$, que representaban menos del $1 \%$ de la prevalencia total de consumo de tabaco).

Dependencia a la nicotina. Para evaluar la dependencia a la nicotina se utilizó el test de Fagerström³ ${ }^{3}$. Las preguntas incluyen: tiempo transcurrido desde que el individuo se despierta hasta que fuma el primer cigarrillo del día (0-3 puntos); dificultad para abstenerse de fumar en lugares donde está prohibido hacerlo (0-1 punto); cigarrillo del día al que le cuesta más renunciar (0-1 punto); número de cigarrillos fumados por día (0-3 puntos); momento del día en que fuma más frecuentemente (0-1 punto); y si fuma aun estando enfermo (0-1 punto). Se describieron los niveles de dependencia a la nicotina como baja (0-4 puntos), media (5 puntos) y alta (6-10 puntos) ${ }^{2}$.

Estadios del cambio: Se consideraron los estadios del cambio del modelo transteórico de Prochaska y DiClemente ${ }^{4,14}$ correspondientes a la fase fumadora: precontemplación, en el que los fumadores no se plantean dejar de fumar en un futuro cercano; contemplación, en el que los fumadores consideran seriamente dejar de fumar durante los próximos 6 meses; y preparación, que describe a los fumadores que desean dejar de fumar en el transcurso del próximo mes y han realizado al menos un intento no exitoso de dejar de fumar en el último año.

\section{Análisis estadístico}

Se calculó la distribución de las variables estudiadas mediante proporciones y sus intervalos de confianza del 95\% (IC 95\%) según sexo, grupos de edad (18-39, 40-59 $y \geq 60$ años), nivel de estudios (primaria o menos que primaria, secundaria y universitaria), edad de inicio del consumo $(<15,15-18$ y $\geq 19$ años) y número de cigarrillos fumados diariamente $(<10,10-19 y \geq 20$ cigarrillos). Se describió gráficamente la distribución de los fumadores de acuerdo a sus puntuaciones en el test de Fagerström. Los análisis fueron realizados con los programas SPSS 15.0 y Excel. 


\section{RESULTADOS}

De los 568 participantes que declararon fumar cigarrillos en el momento de la entrevista (22,5\% de la muestra), el $54,4 \%$ eran hombres, el 57,0\% tenía entre 18-39 años y el 44,5\% tenía estudios secundarios. La mayoría de los fumadores $(56,5 \%)$ comenzaron a fumar entre los 15 y 18 años. El 36,1\% fumaba entre 10 y 19 cigarrillos por día (media de 14,4 cigarrillos; desviación estándar de 9,2).

La mayoría de los fumadores tenía una baja dependencia a la nicotina (Figura 1): el 74,1\% obtuvo puntuaciones en el test de Fagerström entre 0 y 4 . La puntuación media en el test fue de 2,80 (2,97 los hombres y 2,58 las mujeres). En la Tabla 1 se presenta la distribución de los fumadores según su nivel de dependencia a la nicotina, de acuerdo a las variables sociodemográficas y algunas características del consumo. La distribución de los fumadores según sexo a través de los niveles de dependencia era homogénea. Se puede constatar un mayor porcentaje de fumadores con dependencia media y alta entre 40-59 años comparados con los otros grupos de edad. La mayor prevalencia de fumadores con dependencia alta a la nicotina se observó en el grupo con estudios primarios $(22,6 \%)$ y en los que habían comenzado a fumar antes de los 15 años (35,6\%). La mayoría de los fumadores de menos de 10 cigarrillos diarios tenía una baja dependencia a la nicotina, mientras que la proporción de fumadores con dependencia media y alta aumentaba con el consumo de cigarrillos (Tabla 1).
En cuanto a la distribución de los fumadores según los estadios del cambio, el $64,3 \%$ de los fumadores estaban en el estadio de precontemplación, el 25,4\% en el de contemplación, y el $10,4 \%$ en el estadio de preparación, sin diferencias según sexo (Tabla 2). Los fumadores $\geq 60$ años estaban más preparados para dejar de fumar que los fumadores más jóvenes. Aquéllos que comenzaron a fumar antes de los 18 años se encontraban mayoritariamente en el

Figura 1. Distribución de 568 fumadores españoles según sus puntuaciones en el test de Fagerström, 2006.

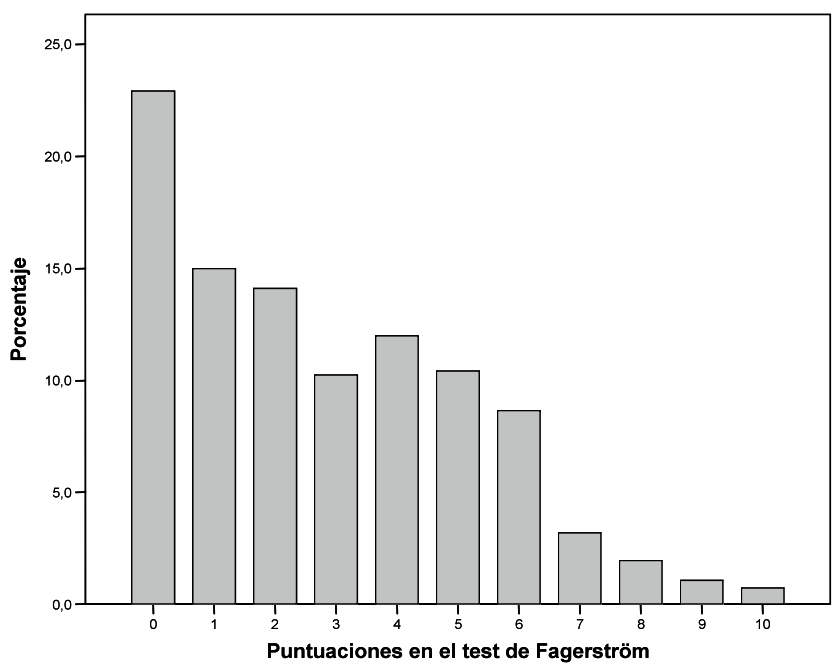

Tabla 1. Prevalencia (\%) e intervalo de confianza del 95\% (IC 95\%) de los niveles de dependencia a la nicotina en 568 fumadores españoles según variables sociodemográficas y algunas características del consumo de tabaco, 2006.

\begin{tabular}{|c|c|c|c|c|c|c|c|}
\hline & \multirow[b]{3}{*}{$\mathrm{n}$} & \multicolumn{6}{|c|}{ Dependencia a la nicotina* } \\
\hline & & \multicolumn{2}{|c|}{ baja } & \multicolumn{2}{|c|}{ media } & \multicolumn{2}{|c|}{ alta } \\
\hline & & $\%$ & IC 95\% & $\%$ & IC 95\% & $\%$ & IC 95\% \\
\hline Total & 568 & 74,1 & $70,5-77,7$ & 10,4 & $7,9-12,9$ & 15,5 & $12,5-18,5$ \\
\hline \multicolumn{8}{|l|}{ Sexo } \\
\hline hombres & 309 & 73,5 & $68,5-78,4$ & 10,4 & $7,0-13,8$ & 16,2 & $12,1-20,3$ \\
\hline mujeres & 259 & 74,9 & $69,6-80,2$ & 10,4 & $6,7-14,1$ & 14,7 & $10,4-19,0$ \\
\hline \multicolumn{8}{|l|}{ Edad } \\
\hline 18-39 años & 324 & 79,6 & $75,2-84,0$ & 8,3 & $5,3-11,3$ & 12,0 & $8,5-15,6$ \\
\hline 40-59 años & 207 & 65,7 & $59,2-72,2$ & 13,5 & $8,9-18,2$ & 20,8 & $15,2-26,3$ \\
\hline$\geq 60$ años & 37 & 73,0 & $57,0-84,6$ & 10,8 & $4,3-24,7$ & 16,2 & $7,7-31,1$ \\
\hline \multicolumn{8}{|l|}{ Estudios } \\
\hline primarios y menos & 195 & 65,1 & $58,4-71,8$ & 12,3 & $7,7-16,9$ & 22,6 & $16,7-28,4$ \\
\hline secundarios & 253 & 79,4 & $74,5-84,4$ & 9,9 & $6,2-13,6$ & 10,7 & $6,9-14,5$ \\
\hline universitarios & 119 & 77,3 & $69,8-84,8$ & 8,4 & $3,4-13,4$ & 14,3 & $8,0-20,6$ \\
\hline \multicolumn{8}{|c|}{ Edad de inicio del consumo } \\
\hline$<15$ años & 87 & 52,9 & $42,4-63,4$ & 11,5 & $4,8-18,2$ & 35,6 & $25,6-45,7$ \\
\hline 15-18 años & 321 & 77,9 & $73,3-82,4$ & 10,3 & $7,0-13,6$ & 11,8 & $8,3-15,4$ \\
\hline$\geq 19$ años & 160 & 78,1 & $71,7-84,5$ & 10,0 & $5,4-14,6$ & 11,9 & $6,9-16,9$ \\
\hline \multicolumn{8}{|l|}{ Número de cigarrillos } \\
\hline$<10$ cigarrillos/dia & 183 & 99,5 & $97,0-99,9$ & 0,5 & $0,1-3,0$ & 0,0 & $0,0-2,1$ \\
\hline 10-19 cigarrillos/día & 205 & 82,9 & $77,8-88,1$ & 10,7 & $6,5-15,0$ & 6,3 & $3,0-9,7$ \\
\hline$\geq 20$ cigarrillos/día & 180 & 38,3 & $31,2-45,4$ & 20,0 & $14,2-25,8$ & 41,7 & $34,5-48,9$ \\
\hline
\end{tabular}

*Según la puntuación en el test de Fagerström: baja: 0-4 puntos; media: 5 puntos; alta: 6-10 puntos. 
Tabla 2. Prevalencia (\%) e intervalo de confianza del 95\% (IC 95\%) de los estadios del cambio en 568 fumadores españoles según variables sociodemográficas y algunas características del consumo de tabaco, 2006.

\begin{tabular}{|c|c|c|c|c|c|c|c|}
\hline & \multirow[b]{3}{*}{$n$} & \multicolumn{6}{|c|}{ Estadios del cambio } \\
\hline & & \multicolumn{2}{|c|}{ precontemplación } & \multicolumn{2}{|c|}{ contemplación } & \multicolumn{2}{|c|}{ preparación } \\
\hline & & $\%$ & IC 95\% & $\%$ & IC 95\% & $\%$ & IC 95\% \\
\hline Total & 568 & 64,3 & $60,3-68,2$ & 25,4 & $21,8-28,9$ & 10,4 & $7,9-12,9$ \\
\hline \multicolumn{8}{|l|}{ Sexo } \\
\hline hombres & 309 & 66,0 & $60,7-71,3$ & 22,7 & $18,0-27,3$ & 11,3 & $7,8-14,9$ \\
\hline mujeres & 259 & 62,2 & $56,3-68,1$ & 28,6 & $23,1-34,1$ & 9,3 & $5,7-12,8$ \\
\hline \multicolumn{8}{|l|}{ Edad } \\
\hline 18-39 años & 324 & 65,1 & $59,9-70,3$ & 24,4 & $19,7-29,1$ & 10,5 & $7,2-13,8$ \\
\hline 40-59 años & 207 & 63,3 & $56,7-69,9$ & 28,0 & $21,9-34,1$ & 8,7 & $4,9-12,5$ \\
\hline$\geq 60$ años & 37 & 62,2 & $46,5-77,8$ & 18,9 & $6,3-31,5$ & 18,9 & $6,3-31,5$ \\
\hline \multicolumn{8}{|l|}{ Estudios } \\
\hline primarios y menos & 195 & 65,1 & $58,4-71,8$ & 26,2 & $20,0-32,3$ & 8,7 & $4,8-12,7$ \\
\hline secundarios & 253 & 63,6 & $57,7-69,6$ & 24,9 & $19,6-30,2$ & 11,5 & $7,5-15,4$ \\
\hline universitarios & 119 & 64,7 & $56,1-73,3$ & 24,4 & $16,7-32,1$ & 10,9 & $5,3-16,5$ \\
\hline \multicolumn{8}{|c|}{ Edad de inicio del consumo } \\
\hline$<15$ años & 87 & 67,8 & $58,0-77,6$ & 17,2 & $9,3-25,2$ & 14,9 & $7,5-22,4$ \\
\hline 15-18 años & 321 & 67,6 & $62,5-72,7$ & 23,4 & $18,7-28,0$ & 9,0 & $5,9-12,2$ \\
\hline$\geq 19$ años & 160 & 55,6 & $47,9-63,3$ & 33,8 & $26,4-41,1$ & 10,6 & $5,9-15,4$ \\
\hline \multicolumn{8}{|l|}{ Número de cigarrillos } \\
\hline$<10$ cigarrillos/día & 183 & 65,0 & $58,1-71,9$ & 23,5 & $17,4-29,6$ & 11,5 & $6,9-16,1$ \\
\hline 10-19 cigarrillos/dia & 205 & 61,5 & $54,8-68,1$ & 29,3 & $23,0-35,5$ & 9,3 & $5,3-13,2$ \\
\hline$\geq 20$ cigarrillos/día & 180 & 66,7 & $59,8-73,6$ & 22,8 & $16,7-28,9$ & 10,6 & $6,1-15,0$ \\
\hline
\end{tabular}

*Según la puntuación en el test de Fagerström: baja: 0-4 puntos; media: 5 puntos; alta: 6-10 puntos.

Tabla 3. Prevalencia (\%) e intervalo de confianza del 95\% (IC 95\%) de los estadios del cambio según los niveles de dependencia a la nicotina en 568 fumadores españoles, 2006.

\begin{tabular}{|c|c|c|c|c|c|c|c|}
\hline \multirow[b]{3}{*}{ Estadios del cambio } & \multirow[b]{3}{*}{$n$} & \multicolumn{6}{|c|}{ Dependencia a la nicotina* } \\
\hline & & \multicolumn{2}{|c|}{ baja } & \multicolumn{2}{|c|}{ media } & \multicolumn{2}{|c|}{ alta } \\
\hline & & $\%$ & IC 95\% & $\%$ & IC 95\% & $\%$ & IC 95\% \\
\hline precontemplación & 365 & 75,1 & $70,6-79,5$ & 10,4 & $7,3-13,5$ & 14,5 & $10,9-18,1$ \\
\hline contemplación & 144 & 74,3 & $67,2-81,4$ & 10,4 & $5,4-15,4$ & 15,3 & $9,4-21,2$ \\
\hline preparación & 59 & 67,8 & $55,9-79,7$ & 10,2 & $2,5-17,9$ & 22,0 & $11,5-32,6$ \\
\hline
\end{tabular}

*Según la puntuación en el test de Fagerström: baja: 0-4 puntos; media: 5 puntos; alta: 6-10 puntos.

estadio de precontemplación, mientras que no se observó un patrón claro en los fumadores en fase de preparación.

En la Tabla 3 se muestra la distribución conjunta de la dependencia a la nicotina y los estadios de cambio de los fumadores. La proporción de fumadores que tenía una dependencia alta fue ligeramente superior en el estadio de preparación (22,0\%) que en los estadios de precontemplación $(14,5 \%)$ y contemplación $(15,3 \%)$, si bien estas diferencias no fueron estadísticamente significativas.

\section{DISCUSIÓN}

Los resultados de este trabajo indican que la mayoría de los fumadores españoles se encuentran en el estadio de precontemplación y tienen una baja dependencia a la nicotina. Los fumadores más dependientes a la nicotina eran aquéllos con estudios primarios, los que iniciaron el consumo de tabaco a temprana edad, y quienes tenían un mayor consumo de cigarrillos. No se observaron diferencias en la distribución de los fumadores según los estadios del cambio y las variables sociodemográficas estudiadas, al igual que en otras poblaciones ${ }^{15-17}$.

Este estudio es el primero que dispone de datos de fumadores a nivel nacional que describen de manera conjunta su nivel de dependencia y su distribución según los estadios del cambio. El trabajo más amplio publicado hasta ahora era el derivado del estudio IBERPOC, Ilevado a cabo durante 1996-1997 en 7 poblaciones de España (Oviedo, Burgos, Cáceres, Madrid, Sevilla, Manlleu y Bilbao) en población adulta entre 40 y 69 años ${ }^{5}$. En dicho estudio, los 1.059 fumadores entrevistados (26\% de los participantes) tenían una puntuación media de 3,4 en el test de Fagerström $(3,7$ los hombres y 2,6 las mujeres) y aproximadamente el 
$39 \%$ de ellos estaba en el estadio de precontemplación, el $58 \%$ en contemplación y aproximadamente el $4 \%$ en fase de preparación para dejar de fumar ${ }^{5}$. Las diferencias que se observan entre estas cifras y las obtenidas en nuestro estudio podrian explicarse por el propio desarrollo de la epidemia del tabaquismo en el período transcurrido entre ambos estudios (10 años), que bien pudiera condicionar un cambio real de la distribución de los fumadores según los estadios del cambio. En estos 10 años, la prevalencia de exfumadores ha aumentado, seguramente a expensas de fumadores en fase contemplativa, por lo que la proporción de precontempladores puede haber aumentado, tal y como muestran los resultados de nuestro estudio. No podemos excluir que parte de las diferencias observadas tengan su origen en diferencias metodológicas entre nuestro estudio y el estudio IBERPOC ${ }^{18}$, tales como la procedencia de la muestra (obtenida de 7 poblaciones españolas), la forma de contacto con los participantes (por correo o por teléfono), la inclusión de pruebas funcionales respiratorias que podria condicionar la participación, y el acotado grupo de edad estudiado. Al restringir los datos de nuestro estudio a los fumadores entre 40 y 69 años, se observa el mismo valor medio en el test de Fagerström que en el estudio IBERPOC, pero no se observan cambios sustanciales en la distribución de los fumadores según los estadios del cambio respecto a toda la muestra $\geq 18$ años $(63,4 \%, 27,2 \%$ y 9,5\% para las fases de precontemplación, contemplación y preparación, respectivamente)

Otros estudios españoles realizados en los últimos 15 años en diferentes poblaciones describen puntuaciones entre 2,3 y 3,8 en el test de Fagerström ${ }^{6,10,19,20}$ y una distribución de fumadores de acuerdo a los estadios del cambio de aproximadamente entre 40 y $69 \%$ en precontemplación, 25 y $46 \%$ en contemplación y 6 y $14 \%$ en preparación $n^{6,19,21,22}$. A pesar de existir cierta variabilidad, se puede constatar que la preparación para dejar de fumar en la población es distinta a la que se observa en el contexto clínico de deshabituación tabáquica, que oscila entre 32 y $66 \%, 5$ y $26 \%$ y 7,6 y $20 \%$ para las fases de precontemplación, contemplación y preparación, respectivamente ${ }^{8,9,11}$. Además, se observan mayores puntuaciones en el test de Fagerström en los fumadores en deshabituación tabáquica, con independencia de que presenten o no alguna patología7,8. Esto podría explicarse porque aquéllos que son más dependientes a la nicotina pueden tener la percepción de menor autocontrol frente al consumo de tabaco y por ello buscan activamente la ayuda para dejar de fumar.

Debido a que gran parte de los estudios realizados a nivel poblacional indican que la mayoría de los fumadores se encuentran en fase de precontemplación ${ }^{6,21,23}$ y tienen una baja dependencia a la nicotina ${ }^{5,6,10}$, las intervenciones a este nivel deberían orientar sus esfuerzos a intentar que los fumadores se muevan hacia fases más avanzadas de los estadios del cambio. Así, se debería intentar motivar a aquellos fumadores que no desean dejar de fumar, y propiciar que los fumadores con una dependencia baja a la nicotina sean capaces de dejar el consumo. Los factores que han sido descritos como los más influyentes en la intención futura de dejar el consumo son: la preocupación por los efectos de la exposición pasiva en los niños, familiares y amigos de los fumadores; la preocupación por el papel modélico de los fumadores entre los niños y jóvenes; y el consejo médico ${ }^{24}$. De esta forma, las actividades de control del tabaquismo a nivel poblacional deberían incluir como mínimo estos aspectos $^{25,26}$.

La descripción de los fumadores de acuerdo al modelo transteórico y al grado de dependencia a la nicotina es útil en la planificación y evaluación de intervenciones poblacionales, así como en el tratamiento individualizado del tabaquismo, pues ayuda a comprender el grado y la naturaleza de la adicción. Estudios derivados de intervenciones clínicas en deshabituación tabáquica han identificado entre los factores que se asocian a la abstinencia el tener bajos niveles de dependencia a la nicotina ${ }^{27-30}$ y encontrarse en estadios del cambio avanzados ${ }^{28-31}$, si bien otros estudios señalan que el test de Fagerström ${ }^{32}$ o los estadios del cambio ${ }^{33}$ no predicen tan claramente la conducta tabáquica. A pesar de ello, se debe tener en cuenta que estos trabajos están realizados en un contexto clínico especializado, en el que es más frecuente que los sujetos estén motivados para dejar de fumar.

Una de las limitaciones de este estudio deriva del uso de un cuestionario, que recoge información autodeclarada del consumo sin un marcador objetivo del mismo, y por tanto está sujeta a un posible sesgo de información que puede afectar a la validez interna del estudio. Sin embargo, la validez de la autodeclaración del consumo de tabaco es muy elevada ${ }^{34,35}$. Otro posible sesgo de información puede generarse debido a la tendencia de los participantes a redondear al alza el consumo de cigarrillos, especialmente entre los grandes fumadores ${ }^{36}$. Otra limitación se deriva del hecho de haber utilizado un directorio de teléfonos fijos para la obtención de la muestra de estudio, considerando que en los últimos años el uso del teléfono móvil se ha ido extendiendo en la población, pudiendo incluso en algunos casos llegar a sustituir el uso del teléfono fijo, lo cual podría afectar a la representatividad geográfica de la muestra. Además, por tratarse de una encuesta telefónica, puede existir un sesgo de información debido a la deseabilidad social, reforzada por la reciente implantación de la Ley 28/2005 en el momento del trabajo de campo. Todo lo anterior podría explicar el hecho que la prevalencia de consumo en nuestro estudio sea ligeramente menor comparada con la obtenida en la Encuesta Nacional de Salud de España del año $2006^{37}$, aunque similar a la de la encuesta del Centro de Investigaciones Sociológicas del año $2006^{38}$.

Por otra parte, la principal fortaleza de este estudio reside en el hecho de disponer de datos provenientes de una muestra representativa de la población general española. Asimismo, los cuestionarios utilizados en este estudio para caracterizar la dependencia y la preparación para dejar de fumar han sido validados y ampliamente utilizados en la literatura internacional ${ }^{39}$. Obtener esta información a nivel poblacional es sencillo y factible, y disponer de ella de manera periódica ayudaría a planificar programas de salud pública orientados a disminuir la prevalencia de fumadores en el estadio de precontemplación y aumentar la de estadios 
más avanzados. Se ha demostrado que la intención de dejar de fumar puede cambiar a través del tiempo, a veces espontáneamente e incluso en cortos períodos de tiempo ${ }^{40}$, con lo cual intervenciones eficaces podrían acelerar este movimiento deseado.

En conclusión, este trabajo indica que la mayoría de los fumadores españoles se encuentra en fase de precontemplación y tiene una baja dependencia a la nicotina. Existe cierta relación entre la preparación para dejar de fumar y la dependencia a la nicotina, dado que los fumadores menos dependientes se concentran en la fase de precontemplación y los más dependientes en la fase de preparación. Los programas de deshabituación deberian ir dirigidos a reducir el nivel de dependencia, ayudar a los fumadores a avanzar a través de los estadios del cambio, $y$, consecuentemente, disminuir la prevalencia de fumadores en la población.

\section{AGRADECIMIENTOS}

Este estudio ha sido parcialmente financiado con fondos de la Comisión Europea (proyecto de la European Network for Smoking Prevention, ref. 2004323), el Instituto de Salud Carlos III del Ministerio de Sanidad (Red Temática de Investigación en Cáncer, ref. RD06/0020/0089), y el Department d'Universitats i Recerca de la Generalitat de Catalunya (ref. 2009 SGR 192).

\section{CONFLICTOS DE INTERESES}

Los autores declaran que no tienen conflictos de intereses.

\section{REFERENCIAS}

1. U.S. Department of Health and Human Services. The health consequences of smoking: a report of the Surgeon General. Atlanta, GA: U.S. Department of Health and Human Services, Centers for Disease Control and Prevention, National Center for Chronic Disease Prevention and Health Promotion, Office on Smoking and Health; 2004.

2. Heatherton TF, Kozlowski LT, Frecker RC, Fagerström KO. The Fagerström Test for Nicotine Dependence: a revision of the Fagerström Tolerance Questionnaire. Br J Addict 1991; 86: 111927

3. Becoña $E$, Vázquez FL. The Fagerström Test for Nicotine Dependence in a Spanish sample. Psychol Rep 1998; 83: 14558.

4. Prochaska JO, DiClemente CC, Norcross JC. In search of how people change. Applications to addictive behaviors. Am Psychol 1992; 47: 1102-14.

5. Jiménez Ruiz CA, Fernando MJ, Sobradillo V, Gabriel R, Miravitlles $M$, Fernández-Fau $L$ et al. Prevalencia y actitudes sobre tabaquismo en población mayor de 40 años. Arch Bronconeumol 2000; 36: 241-4.
6. Bellido Casado J, Martín Escudero JC, Duenas Laita A, Mena Martín FJ, Arzúa Mouronte D, Simal Blanco F. Hábito tabáquico en una población general: descripción de la prevalencia, grado de consolidación y fase de abandono. Arch Bronconeumol 2001; 37: 75-80.

7. Morchón S, Blasco JA, Rovira A, Arias CN, Ramón JM. Efectividad de una intervención de deshabituación tabáquica en pacientes con patologia cardiovascular. Rev Esp Cardiol 2001; 54: 1271-6.

8. Dueñas Herrero RM, Aleu Bover M, González Tejón I, San Molina L, Salavert Jiménez J, Arranz Martí B. Impact of medical counseling to quit smoking during the process of dehabituation in patients with mental illness. Actas Esp Psiquiatr 2004; 32: 287-92.

9. Peris Cardells R, Rico Salvador I, Herrera de Pablo P, Pérez Jiménez A, Sánchez-Tóril López F, Pérez Fernández JA. Estudio demográfico del tabaquismo en el Área de Salud 5 de Valencia. Arch Bronconeumol 2004; 40: 160-5.

10. Pérez-Ríos M, Santiago-Pérez MI, Alonso B, Malvar A, Hervada $X$, de Leon J. Fagerstrom test for nicotine dependence vs heavy smoking index in a general population survey. BMC Public Health 2009; 9: 493.

11. Albareda M, Sánchez L, González J, Viguera J, Mestrón A, Vernet A et al. Results of the application of the American Diabetes Association guidelines regarding tobacco dependency in subjects with diabetes mellitus. Metabolism 2009; 58: 1234-8.

12. Fernandez E, Lopez MJ, Nebot M, ETS EuroSurvey Working Group. Exposure to environmental tobacco smoke in the general population of 6 European countries, 2006. Book of abstracts. Basilea: European Conference on Tobacco or Health 2006; p. 77.

13. Lushchenkova O, Fernandez E, Lopez MJ, Fu M, MartinezSanchez JM, Nebot M et al. Exposición al humo ambiental de tabaco en población adulta no fumadora en España tras la Ley de medidas sanitarias frente al tabaquismo. Rev Esp Cardiol 2008; 61: 687-94.

14. Prochaska JO, DiClemente CC. Stages and processes of selfchange of smoking: toward an integrative model of change. J Consult Clin Psychol 1983; 51: 390-5.

15. DiClemente CC, Prochaska JO, Fairhurst SK, Velicer WF, Velasquez MM, Rossi JS. The process of smoking cessation: an analysis of precontemplation, contemplation, and preparation stages of change. J Consult Clin Psychol 1991; 59: 295-304.

16. John U, Meyer $C$, Rumpf HJ, Hapke U. Relation among stage of change, demographic characteristics, smoking history, and nicotine dependence in an adult German population. Prev Med 2003; 37: 368-74.

17. Thyrian JR, Panagiotakos DB, Polychronopoulos E, West $\mathrm{R}$, Zatonski $\mathrm{W}$, John $\mathrm{U}$. The relationship between smokers' motivation to quit and intensity of tobacco control at the population level: a comparison of five European countries. BMC Public Health 2008; 8: 2.

18. Miravitlles M, Sobradillo V, Villasante $C$, Gabriel R, Masa $J F$, Jiménez CA et al. Estudio epidemiológico de la EPOC en España (IBERPOC): reclutamiento y trabajo de campo. Arch Bronconeumol 1999; 35: 152-8.

19. de Granda-Orive Jl, Peña-Miguel T, Jiménez-Ruiz DC, SolanoReina DS, Martínez-Albiach JM, Escobar-Sacristán J et al. Distribution of stages of change in smoking behavior in a group of young smokers (transtheoretical model). Mil Med 2004; 169: 972-5. 
20. Molina AJ, Fernández D, Delgado M, Martín V. Sensitivity and specificity of a self-administered questionnaire of tobacco use; including the Fagerstrom test. Int J Nurs Stud 2010; 47: 181-9.

21. Becoña E. Estadios de cambio en la conducta de fumar: Su relevancia en el tratamiento de fumadores. Prev Tab 2000; 2: 106-12.

22. Font-Mayolas S, Planes M, Gras ME. Caracteristicas de la conducta de fumar en función de la fase de abandono en una muestra de personal de la Universidad de Girona. Prev Tab 2004; 6: 60-7.

23. Valverde A, Saltó E, Ortega G, Medina A, Brugulat P, Tresseras R et al. Los fumadores que quieren dejarlo: estadios de cambio en la población de Cataluña. Gac Sanit 2007; 21(Supl 2): 25.

24. Boyle P, Gandini S, Robertson C, Zatonski W, Fagerstrom K, Slama K et al. Characteristics of smokers' attitudes towards stopping: survey of 10,295 smokers in representative samples from 17 European countries. Eur J Public Health 2000; 10: 5-14.

25. Stead LF, Bergson G, Lancaster T. Asesoramiento médico para el abandono del hábito de fumar (Revisión Cochrane traducida). En: La Biblioteca Cochrane Plus, 2008 Número 4. Oxford: Update Software Ltd. Disponible en: http://www.update-software.com. (Traducida de The Cochrane Library, 2008 Issue 3. Chichester, UK: John Wiley \& Sons, Ltd.); 2008.

26. Lai DTC, Cahill K, Qin Y, Tang JL. Motivational interviewing for smoking cessation. Cochrane Database of Systematic Reviews 2010; Issue 1. Art. No.: CD006936. D0I: 10.1002/14651858. CD006936.pub2.

27. Norregaard J, Tonnesen P, Petersen L. Predictors and Reasons for Relapse in Smoking Cessation with Nicotine and Placebo Patches. Prev Med 1993; 22: 261-71.

28. Ferguson JA, Patten CA, Schroeder DR, Offord KP, Eberman KM, Hurt RD. Predictors of 6-month tobacco abstinence among 1224 cigarette smokers treated for nicotine dependence. Addict Behav 2003; 28: 1203-18.

29. Rohren $C L$, Croghan IT, Hurt RD, Offord KP, Marusic Z, Mcclain FL. Predicting Smoking Cessation Outcome in a Medical Center from Stage of Readiness: Contemplation Versus Action. Prev Med 1994; 23: 335-44.

30. Breitling LP, Twardella D, Raum E, Brenner H. Situational temptation scores and smoking cessation in general care. Psychol Addict Behav 2009; 23: 362-7.

31. Dijkstra A, Roijackers J, De VH. Smokers in four stages of readiness to change. Addict Behav 1998; 23: 339-50.

32. Andersen $\mathrm{S}$. Adding addiction to the transtheoretical model for smoking cessation. Addict Behav 2007; 32: 1099-104.

33. Carlson LE, Taenzer P, Koopmans J, Casebeer A. Predictive value of aspects of the Transtheoretical Model on smoking cessation in a community-based, large-group cognitive behavioral program. Addict Behav 2003; 28: 725-40.

34. Patrick DL, Cheadle A, Thompson DC, Diehr P, Koepsell T, Kinne $S$. The validity of self-reported smoking: a review and metaanalysis. Am J Public Health 1994; 84: 1086-93.

35. Gorber SC, Schofield-Hurwitz S, Hardt J, Levasseur G, Tremblay $M$. The accuracy of self-reported smoking: a systematic review of the relationship between self-reported and cotinine-assessed smoking status. Nicotine Tob Res 2009; 11: 12-24.

36. Klesges RC, Debon M, Ray JW. Are self-reports of smoking rate biased? Evidence from the Second National Health and
Nutrition Examination Survey. J Clin Epidemiol 1995; 48: 122533.

37. Ministerio de Sanidad y Consumo, Instituto Nacional de Estadística. Encuesta Nacional de Salud de España. Disponible en: http://www.msc.es/estadEstudios/ estadisticas/encuestaNacional/ense.htm [fecha de acceso: 20 de diciembre de 2000).

38. Centro de Investigaciones Sociológicas. Tabaquismo y nueva normativa antitabaco, 2006. Estudio 2665. Madrid: CIS; 2006.

39. International Agency for Research on Cancer. IARC Handbooks of Cancer Prevention, Tobacco Control, Vol. 12: Methods for evaluating tobacco control policies. Lyon, France: International Agency for Research on Cancer; 2008.

40. Hughes JR, Keely JP, Fagerstrom KO, Callas PW. Intentions to quit smoking change over short periods of time. Addict Behav 2005; 30: 653-62. 
